\title{
Klein-Gordon Equation with Superintegrable Systems: Kepler-Coulomb, Harmonic Oscillator, and Hyperboloid
}

\author{
V. Mohammadi, S. Aghaei, and A. Chenaghlou \\ Department of Physics, Faculty of Science, Sahand University of Technology, P.O. Box 51335-1996, Tabriz, Iran \\ Correspondence should be addressed to A. Chenaghlou; a.chenaghlou@sut.ac.ir
}

Received 27 July 2015; Revised 26 September 2015; Accepted 15 October 2015

Academic Editor: Thomas Rössler

Copyright (c) 2015 V. Mohammadi et al. This is an open access article distributed under the Creative Commons Attribution License, which permits unrestricted use, distribution, and reproduction in any medium, provided the original work is properly cited. The publication of this article was funded by SCOAP S $^{3}$

\begin{abstract}
We study the two-dimensional Klein-Gordon equation with spin symmetry in the presence of the superintegrable potentials. On Euclidean space, the SO(3) group generators of the Schrödinger-like equation with the Kepler-Coulomb potential are represented. In addition, by Levi-Civita transformation, the Schrödinger-like equation with harmonic oscillator which is dual to the KeplerCoulomb potential and the SU(2) group generators of associated system are studied. Also, we construct the quadratic algebra of the hyperboloid superintegrable system. Then, we obtain the corresponding Casimir operators and the structure functions and the relativistic energy spectra of the corresponding quasi-Hamiltonians by using the quadratic algebra approach.
\end{abstract}

\section{Introduction}

In the relativistic quantum mechanics, the Dirac and the Klein-Gordon (KG) equations are the most appropriate wave equations to describe the dynamics of the particles with spin $1 / 2$ and 0 , respectively. The Dirac and KG equations with scalar and vector potentials of equal magnitude (SVPEM), with the identical or opposite signs, have a spin or pseudospin symmetry [1-3]. These relativistic systems with spin or pseudospin symmetry are reduced to a Schrödinger-like equation which is called quasi-Hamiltonian [4]. So, one can use the nonrelativistic mathematical techniques to investigate the relativistic systems with spin or pseudospin symmetry.

Recently, many works have been done in connection with the Dirac and the KG equations with SVPEM [1-21]. Quadratic algebra approach is a mathematical technique that was constructed by Daskaloyannis in the nonrelativistic quantum mechanics [22]. He considered the quadratic integrals of the motion of the superintegrable systems in the twodimensional space and obtained the corresponding quadratic algebras. Then, by realization of this quadratic algebra in terms of the deformed oscillator algebra, he derived the structure function and the energy spectrum. The relativistic systems with SVPEM are reduced to a Schrödinger-like equation. So, the quadratic algebra approach can be employed in the relativistic quantum mechanics $[1,6,7,9,13,23-27]$. In this paper, we want to apply the quadratic algebra approach to solve the KG equation with SVPEM in the presence of some superintegrable potentials on the two-dimensional hyperboloid and Euclidean space. Consequently, by realization of this algebra with the deformed oscillator algebra [22], the energy eigenvalues may be obtained.

In what follows, it is shown that the KG equation with SVPEM is reduced to the Schrödinger-like equation. We investigate 2-dimensional Klein-Gordon equation that can be reduced to superintegrable systems. Indeed only in the case of spin or pseudospin symmetries, the $2 \mathrm{D}$ KG equation is converted to the Schrodinger-like equation. In other words, the quasi-Hamiltonians on both Euclidean plane and Hermitian hyperboloid are formulated for the relativistic systems. In Section 3, the realization of the quadratic algebra in terms of the deformed oscillator algebra is reviewed [22, 28-30]. In Section 4, we consider the quasi-Hamiltonians with harmonic oscillator and the Kepler-Coulomb superintegrable potentials which are dual to each other by Levi-Civita transformation [31]. The duality of these two superintegrable systems is shown in both relativistic and nonrelativistic quantum mechanics $[32,33]$. Furthermore, we investigate 
the quasi-Hamiltonian with a hyperboloid superintegrable potential [34]. In fact, superintegrable systems on the twodimensional hyperboloid have attracted a considerable attention and have been investigated in [35-37] which contain an introduction, a development, and motivation. Also, in spaces of constant negative curvature like hyperboloid, there are generally more orthogonal coordinate systems which separate the Schrödinger equation compared to spaces of flat or constant positive curvature. By deriving the integrals of motion for the superintegrable systems, we calculate the corresponding quadratic algebras. Then, the quadratic algebra approach is used to calculate the Casimir operators, structure functions of the superintegrable systems, and relativistic energy spectra. The results are discussed in the last section.

\section{Klein-Gordon Equation with Spin Symmetry}

The Klein-Gordon wave equation describes the spinless particles. The KG equation is given by

$$
\left(\partial_{\mu} \partial^{\mu}+\frac{m^{2} c^{2}}{\hbar^{2}}\right) \psi(t, \mathbf{r})=0
$$

where

$$
\partial_{\mu} \partial^{\mu}=\frac{1}{c^{2}} \frac{\partial^{2}}{\partial t^{2}}-\nabla^{2}
$$

The time-independent KG equation takes the following form:

$$
\left\{c^{2} P^{2}+\left(m c^{2}+S(\mathbf{r})\right)^{2}-(\mathscr{E}-V(\mathbf{r}))^{2}\right\} \psi(\mathbf{r})=0,
$$

where $S(\mathbf{r})$ and $V(\mathbf{r})$ are the scalar and vector potentials, respectively, and $\mathscr{E}$ is the relativistic energy. In the special case of the spin symmetry, the scalar and vector potentials have equal magnitude [7]; that is,

$$
S(\mathbf{r})=+V(\mathbf{r})=\frac{\widetilde{V}(\mathbf{r})}{2} .
$$

Thus, the KG equation (3) becomes

$$
\left\{c^{2} P^{2}+\left(m c^{2}+\mathscr{E}\right) \widetilde{V}(\mathbf{r})-\left(\mathscr{E}^{2}-m^{2} c^{4}\right)\right\} \psi(\mathbf{r})=0 .
$$

In this case, the Klein-Gordon equation is reduced to a Schrödinger-like equation which is called the quasi-Hamiltonian. Therefore, the solution of the quasi-Hamiltonian with no spin-dependence leads to the relativistic energy eigenvalues. If we set

$$
\begin{aligned}
2 \widetilde{m} & =\frac{\mathscr{E}}{c^{2}}+m, \\
E & =\mathscr{E}-m c^{2}
\end{aligned}
$$

$$
\text { for } \mathscr{E} \neq m c^{2},
$$

then we can rewrite (5) as a Schrödinger-like equation:

$$
\left(\frac{P^{2}}{2 \widetilde{m}}+\widetilde{V}(\mathbf{r})\right) \psi(\mathbf{r})=\widetilde{H} \psi(\mathbf{r})=E \psi(\mathbf{r}),
$$

where $\widetilde{H}=\left(P^{2} / 2 \widetilde{m}+\widetilde{V}(\mathbf{r})\right)$ is the quasi-Hamiltonian and $E$ is nonrelativistic energy. Also, the quasi-Hamiltonian $\widetilde{H}$ is applicable to the pseudospin symmetry case (i.e., $S(\mathbf{r})=$ $-V(\mathbf{r}))$ by making some corrections.

In this paper, we investigate the superintegrable systems on the two-dimensional Euclidean space and on the hyperboloid. The Hamiltonian $\widetilde{H}$ holds for the Euclidean plane, whereas we shall introduce the Laplace-Beltrami operator for the Hermitian hyperboloid case; that is,

$$
\Delta_{\mathrm{LB}}=-M_{1}^{2}+K_{2}^{2}+K_{3}^{2} \text {, }
$$

where

$$
\begin{gathered}
M_{1}=\omega_{2} \partial_{\omega_{3}}-\omega_{3} \partial_{\omega_{2}}, \\
K_{2}=\omega_{1} \partial_{\omega_{3}}+\omega_{3} \partial_{\omega_{1}}, \\
K_{3}=\omega_{1} \partial_{\omega_{2}}+\omega_{2} \partial_{\omega_{1}},
\end{gathered}
$$

and these operators generate the Lie algebra so(2, 1) [38]:

$$
\begin{gathered}
{\left[M_{1}, K_{2}\right]=K_{3},} \\
{\left[K_{3}, K_{2}\right]=M_{1},} \\
{\left[K_{3}, M_{1}\right]=K_{2} .}
\end{gathered}
$$

The Cartesian coordinates $\omega_{1}, \omega_{2}$, and $\omega_{3}$ are the characterization parameters of the $2 \mathrm{D}$ hyperboloid which satisfy $\omega_{1}^{2}-\omega_{2}^{2}-\omega_{3}^{2}=1$ and $\omega_{1}>1$ (i.e., we consider just upper sheet of double-sheet hyperboloid). Therefore, the KG equation on the two-dimensional Hermitian hyperboloid $(\mathrm{HH}(2))$ with considering SVPEM could be easily constructed by substituting the Laplace-Beltrami operator in (7); that is

$$
\left(\frac{-\hbar^{2}}{2 \widetilde{m}} \Delta_{\mathrm{LB}}+\widetilde{V}(\boldsymbol{\omega})\right) \psi(\boldsymbol{\omega})=\widetilde{H} \psi(\boldsymbol{\omega})=E \psi(\boldsymbol{\omega}),
$$

where $\widetilde{H}=\left(-\hbar^{2} \Delta_{\mathrm{LB}} / 2 \widetilde{m}+\widetilde{V}(\boldsymbol{\omega})\right)$ is the quasi-Hamiltonian on $\mathrm{HH}(2)$.

\section{Quadratic Algebra Approach}

The quadratic algebra for the two-dimensional superintegrable systems has been investigated by Daskaloyannis [22]. Let us briefly review the results of the 2-dimensional nonrelativistic quantum superintegrable systems with second-order integrals of motion and their associated representation of the quadratic algebras which are determined by using deformed oscillator techniques.

If $A$ and $B$ are the second-order integrals of motion of Hamiltonian $H$, then

$$
\begin{aligned}
& {[H, A]=0,} \\
& {[H, B]=0 .}
\end{aligned}
$$

These generators satisfy the following commutation relations:

$$
\begin{aligned}
& {[A, B]=C,} \\
& {[A, C]=\alpha A^{2}+\gamma\{A, B\}+\delta A+\epsilon B+\zeta,} \\
& {[B, C]=a A^{2}-\gamma B^{2}-\alpha\{A, B\}+d A-\delta B+z,}
\end{aligned}
$$


where $\delta, \epsilon, \zeta, d$, and $z$ are the structure constants which are the polynomials of the Hamiltonian $H$. Also, $\{A, B\}$ represents the anticommutation between two operators. Moreover, the Casimir operator could be obtained by the following relation:

$$
\begin{aligned}
K= & C^{2}-\alpha\left\{A^{2}, B\right\}-\gamma\left\{A, B^{2}\right\}+(\alpha \gamma-\delta)\{A, B\} \\
& +\left(\gamma^{2}-\epsilon\right) B^{2}+(\gamma \delta-2 \zeta) B+\frac{2 a}{3} A^{3} \\
& +\left(d+\frac{a \gamma}{3}+\alpha^{2}\right) A^{2}+\left(\frac{a \epsilon}{3}+\alpha \delta+2 z\right) A .
\end{aligned}
$$

Obviously

$$
\begin{aligned}
& {[K, A]=0,} \\
& {[K, B]=0,} \\
& {[K, C]=0 .}
\end{aligned}
$$

Thus, the Casimir operator $K$ can only be written in terms of the Hamiltonian $H$. On the other hand, a deformed oscillator algebra satisfies

$$
\begin{aligned}
{\left[\mathcal{N}, b^{\dagger}\right] } & =b^{\dagger}, \\
{[\mathcal{N}, b] } & =-b, \\
b^{\dagger} b & =\Phi(\mathcal{N}), \\
b b^{\dagger} & =\Phi(\mathcal{N}+1),
\end{aligned}
$$

where the "well behaved" real function $\Phi(x)$ is called the structure function that satisfies the boundary conditions:

$$
\begin{aligned}
\Phi(E, \mathcal{N}=0)=0, & \\
\Phi(E, \mathcal{N}=p+1)=0, & \forall x>0: \Phi(x)>0 .
\end{aligned}
$$

These conditions lead to a Fock space of dimension $p+1$ which is defined by

$$
\begin{aligned}
H|E, n\rangle & =E|E, n\rangle, \\
\mathcal{N}|E, n\rangle & =n|E, n\rangle, \\
b^{\dagger}|E, n\rangle & =\sqrt{\Phi(E, n+1)}|E, n+1\rangle, \quad n=0,1, \ldots, \\
b|E, n\rangle & =\sqrt{\Phi(E, n)}|E, n-1\rangle, \quad n=1,2, \ldots, \\
b|E, 0\rangle & =0 .
\end{aligned}
$$

In this stage, the quadratic algebra (13) should be realized in terms of the deformed oscillator algebra (16). By considering the form of $A$ and $B$,

$$
\begin{aligned}
& A=A(\mathcal{N}), \\
& B=b(\mathcal{N})+b^{\dagger} \rho(\mathcal{N})+\rho(\mathcal{N}) b,
\end{aligned}
$$

there are two cases.
Case $1(\gamma \neq 0)$.

$$
\begin{aligned}
A(\mathcal{N}) & =\frac{\gamma}{2}\left((\mathcal{N}+u)^{2}-\frac{1}{4}-\frac{\epsilon}{\gamma^{2}}\right), \\
b(\mathcal{N}) & =-\frac{\alpha\left((\mathcal{N}+u)^{2}-1 / 4\right)}{4}+\frac{\alpha \epsilon-\gamma}{2 \gamma^{2}} \\
- & \frac{\alpha \epsilon^{2}-2 \delta \epsilon \gamma+4 \gamma^{2} \zeta}{4 \gamma^{4}} \frac{1}{\left((\mathcal{N}+u)^{2}-1 / 4\right)},
\end{aligned}
$$

$\rho(\mathcal{N})$

$$
\begin{aligned}
& =\frac{1}{\left(3 \times 2^{12} \times \gamma^{8}(\mathcal{N}+u)(1+\mathcal{N}+u)(1+2(\mathcal{N}+u))^{2}\right)}, \\
& \Phi(\mathcal{N})=-3072 \gamma^{6} K(-1+2(\mathcal{N}+u))^{2}-48 \gamma^{6}\left(\alpha^{2} \epsilon-\alpha \delta \gamma\right. \\
& \left.+a \epsilon \gamma-d \gamma^{2}\right)(-3+2(\mathcal{N}+u))(-1+2(\mathcal{N}+u))^{4}(1 \\
& +2(\mathcal{N}+u))+\gamma^{8}\left(3 \alpha^{2}+4 a \gamma\right)(-3+2(\mathcal{N}+u))^{2}(-1 \\
& +2(\mathcal{N}+u))^{4}(1+2(\mathcal{N}+u))^{2}+768\left(\alpha \epsilon^{2}-2 \delta \epsilon \gamma\right. \\
& \left.+4 \gamma^{2} \zeta\right)^{2}+32 \gamma^{4}(-1+2(\mathcal{N}+u))^{2}(-1-12(\mathcal{N}+u) \\
& \left.+12(\mathcal{N}+u)^{2}\right)\left(3 \alpha^{2} \epsilon^{2}-6 \alpha \delta \epsilon \gamma+2 a \epsilon^{2} \gamma+2 \delta^{2} \gamma^{2}\right. \\
& \left.-4 d \epsilon \gamma^{2}+8 \gamma^{3} z+4 \alpha \gamma^{2} \zeta\right)-256 \gamma^{2}(-1+2(\mathcal{N}+u))^{2} \\
& +\left(3 \alpha^{2} \epsilon^{2}\right. \\
& -9 \alpha \delta \epsilon^{2} \gamma+a \epsilon^{3} \gamma+6 \delta^{2} \epsilon \gamma^{2}-3 d \epsilon^{2} \gamma^{2}+2 \delta^{2} \gamma^{4}+2 d \epsilon \gamma^{4} \\
& \left.+12 \epsilon \gamma^{3} z-4 \gamma^{5} z+12 \alpha \epsilon \gamma^{2} \zeta-12 \delta \gamma^{3} \zeta+4 \alpha \gamma^{4} \zeta\right) .
\end{aligned}
$$

Case $2(\gamma=0, \epsilon \neq 0$ and $\epsilon>0)$.

$$
\begin{aligned}
& A(\mathcal{N})=\sqrt{\epsilon}(\mathcal{N}+u), \\
& b(\mathcal{N})=-\alpha(\mathcal{N}+u)^{2}-\frac{\delta}{\sqrt{\epsilon}}(\mathcal{N}+u)-\frac{\zeta}{\epsilon}, \\
& \rho(\mathcal{N})=1, \\
& \Phi(\mathcal{N})=+\frac{1}{4}\left(-\frac{K}{\epsilon}-\frac{z}{\sqrt{\epsilon}}-\frac{\delta}{\sqrt{\epsilon}} \frac{\zeta}{\epsilon}+\frac{\zeta^{2}}{\epsilon^{2}}\right)-\frac{1}{12}(3 d \\
& -a \sqrt{\epsilon}-3 \alpha \frac{\delta}{\sqrt{\epsilon}}+3\left(\frac{\delta}{\sqrt{\epsilon}}\right)^{2}-6 \frac{z}{\sqrt{\epsilon}}+6 \alpha \frac{\zeta}{\epsilon} \\
& \left.-6 \frac{\delta}{\sqrt{\epsilon}} \frac{\zeta}{\epsilon}\right)(\mathcal{N}+u)+\frac{1}{4}\left(\alpha^{2}+d-a \sqrt{\epsilon}-3 \alpha \frac{\delta}{\sqrt{\epsilon}}\right. \\
& \left.+\left(\frac{\delta}{\sqrt{\epsilon}}\right)^{2}+2 \alpha \frac{\zeta}{\epsilon}\right)(\mathcal{N}+u)^{2}-\frac{1}{6}\left(3 \alpha^{2}-a \sqrt{\epsilon}\right. \\
& \left.-3 \alpha \frac{\delta}{\sqrt{\epsilon}}\right)(\mathcal{N}+u)^{3}+\frac{1}{4} \alpha^{2}(\mathcal{N}+u)^{4} .
\end{aligned}
$$




\section{Quasi-Hamiltonian with Quantum Superintegrable Systems}

We introduced the Schrödinger-like equation and quasiHamiltonian in Section 2. The generators of Lie group $L_{i}$ which commute with the quasi-Hamiltonian $\widetilde{H}$ in the two dimensions (i.e., $\left[\widetilde{H}, L_{i}\right]=0$ ) are the quadratic integrals of motion that satisfy the quadratic algebra (13). In fact, the potential $\widetilde{V}(\mathbf{r})$ in the quasi-Hamiltonian $\widetilde{H}$ refers to the twodimensional superintegrable potential. Thus, we can use the quadratic algebra approach to find the structure function $\Phi$ and consequently the energy eigenvalue $E$ of the quasiHamiltonian $\widetilde{H}$ just by substituting $E$ and $\widetilde{H}$ in the boundary conditions given by (17) and doing some straightforward algebraic calculations. Subsequently, by means of (6), we can obtain the relativistic energy spectrum $\mathscr{E}$ of the particle which was described by the Klein-Gordon equation with SVPEM.

\subsection{Dual Superintegrable Systems on the}

Two-Dimensional Euclidean Space

4.1.1. Kepler-Coulomb Superintegrable System. Let us consider the motion of the particle on a two-dimensional Euclidean space. Thus the form of the two-dimensional quasiHamiltonian in the presence of the well-known KeplerCoulomb potential in the Euclidean space is given by

$$
\widetilde{H}=\frac{1}{2 \widetilde{m}}\left(P_{x}^{2}+P_{y}^{2}\right)+\frac{\kappa}{\sqrt{x^{2}+y^{2}}},
$$

where $\kappa$ is a constant. In this case, the two second-order integrals of the motion are

$$
\begin{aligned}
& A=\frac{1}{2}\left\{\left(x P_{y}-y P_{x}\right), P_{x}\right\}-\widetilde{m} y \frac{\kappa}{\sqrt{x^{2}+y^{2}}}, \\
& B=\frac{1}{2}\left\{\left(x P_{y}-y P_{x}\right), P_{y}\right\}+\widetilde{m} x \frac{\kappa}{\sqrt{x^{2}+y^{2}}} .
\end{aligned}
$$

Using the quadratic algebra (13) with the above constants of motion, one may yield

$$
\begin{aligned}
& {[A, B]=-i 2 \widetilde{m} \hbar\left(x P_{y}-y P_{x}\right) \widetilde{H},} \\
& {[A, C]=-2 \widetilde{m} \hbar^{2} B \widetilde{H},} \\
& {[B, C]=+2 \widetilde{m} \hbar^{2} A \widetilde{H},}
\end{aligned}
$$

where $C=[A, B]$. Note that $A, B$, and $C$ constitute the generators of $\mathrm{SO}(3)$ Lie symmetry group after normalization. The structure constants are

$$
\epsilon=-d=-2 \widetilde{m} \hbar^{2} \widetilde{H}
$$

however, the other structure constants vanish. For this system, the Casimir operator is given by

$$
K=\widetilde{m}^{2} \hbar^{4} \widetilde{H}^{2}+2 \widetilde{m}^{3} \hbar^{2} \kappa^{2} \widetilde{H} .
$$

In this case $\gamma=0$ and $\epsilon>0$. So, we should use (22) for calculating the structure function. It yields

$$
\Phi(E, \mathcal{N})=\frac{1}{4} \widetilde{m}\left\{\widetilde{m} \kappa^{2}+2 E \hbar^{2}\left(\mathcal{N}+u-\frac{1}{2}\right)^{2}\right\}
$$

Thereby, the boundary conditions (17) imply

$$
u=\frac{1}{2}+\left(\frac{-2 \widetilde{m} \kappa^{2}}{E \hbar^{2}}\right)^{1 / 2}
$$

and, consequently, energy eigenvalues of the quasi-Hamiltonian $\widetilde{H}$ are given by

$$
E=-\frac{2 \widetilde{m} \kappa^{2}}{(p+1)^{2} \hbar^{2}}, \quad p=0,2,4, \ldots
$$

Finally, we obtain the relativistic energy spectrum of the Klein-Gordon equation with SVPEM in the presence of the Kepler-Coulomb superintegrable potential as

$$
\mathscr{E}=m c^{2} \frac{-\kappa^{2}+c^{2} \hbar^{2}(p+1)^{2}}{\kappa^{2}+c^{2} \hbar^{2}(p+1)^{2}} .
$$

4.1.2. Harmonic Oscillator Superintegrable System. Consider the Schrödinger-like equation (7) associated with the quasiHamiltonian (23). Now by using the Levi-Civita transformation which is written as [31]

$$
\left(\begin{array}{l}
x \\
y
\end{array}\right)=\left(\begin{array}{cc}
u_{1} & -u_{2} \\
u_{2} & u_{1}
\end{array}\right)\left(\begin{array}{l}
u_{1} \\
u_{2}
\end{array}\right)
$$

the Schrödinger-like equation of the Kepler-Coulomb system turns into

$$
\left(\frac{1}{8 \widetilde{m}}\left(P_{u_{1}}^{2}+P_{u_{2}}^{2}\right)-E u^{2}\right) \psi=-\kappa \psi
$$

where $u^{2}=u_{1}^{2}+u_{2}^{2}=\left(x^{2}+y^{2}\right)^{1 / 2}$. Using the definitions [33],

$$
\begin{aligned}
\widetilde{\mu} & =4 \widetilde{m}, \\
\widetilde{\omega}^{2} & =-\frac{E}{2 \widetilde{m}}, \\
\varepsilon & =-\kappa,
\end{aligned}
$$

(33) could be written as follows:

$$
\left(\frac{1}{2 \widetilde{\mu}}\left(P_{u_{1}}^{2}+P_{u_{2}}^{2}\right)+\frac{1}{2} \tilde{\mu} \widetilde{\omega}^{2} u^{2}\right) \psi=\widetilde{H} \psi=\varepsilon \psi
$$

in which $\varepsilon$ is the energy eigenvalue of the $2 \mathrm{D}$ harmonic oscillator. Equation (35) is the Schrödinger-like equation of the two-dimensional harmonic oscillator superintegrable potential which is identical to the Kepler-Coulomb superintegrable potential via the Levi-Civita transformation (32). 
The energy $\varepsilon$ and the cyclic frequency $\omega$ are two characterization parameters of an oscillator which are related to each other by $\varepsilon=(N+D / 2) \hbar \omega$ in the quantum mechanics $(N=$ $0,1,2, \ldots$, and $D$ refers to dimension of the configuration space of the oscillator). In a standard situation, $\omega$ is fixed, whereas the energy is quantized. In a nonstandard situation, the energy is fixed and frequency $\omega$ is quantized. Also it could be interpreted that the energy eigenvalues of a physical system depend on the frequency of the oscillator by some transformations. Hence the physical system is identical to the oscillator and the energy spectra of both systems are related to each other by the same transformations.

The quasi-Hamiltonian of the Schrodinger-like equation (35) reads

$$
\widetilde{H}=\frac{1}{2 \widetilde{\mu}}\left(P_{u_{1}}^{2}+P_{u_{2}}^{2}\right)+\frac{1}{2} \widetilde{\mu} \widetilde{\omega}^{2}\left(u_{1}^{2}+u_{2}^{2}\right),
$$

where $\tilde{\mu} \widetilde{\omega}^{2}=M \omega^{2}$ in analogy with the nonrelativistic case. The two quadratic integrals of motion of this system are given by

$$
\begin{aligned}
& A=\left(P_{u_{1}}^{2}-P_{u_{2}}^{2}\right)+\tilde{\mu}^{2} \widetilde{\omega}^{2}\left(u_{1}^{2}-u_{2}^{2}\right), \\
& B=P_{u_{1}} P_{u_{2}}+\widetilde{\mu}^{2} \widetilde{\omega}^{2} u_{1} u_{2} .
\end{aligned}
$$

$A$ and $B$ satisfy the following commutation relations:

$$
\begin{aligned}
& {[A, B]=i 4 \widetilde{\mu}^{2} \widetilde{\omega}^{2} \hbar\left(u_{1} P_{u_{2}}-u_{2} P_{u_{1}}\right),} \\
& {[A, C]=16 \widetilde{\mu}^{2} \widetilde{\omega}^{2} \hbar^{2} B,} \\
& {[B, C]=-4 \widetilde{\mu}^{2} \widetilde{\omega}^{2} \hbar^{2} A,}
\end{aligned}
$$

where $C=[A, B]$. Note that, after normalization, $A, B$, and $C$ constitute the generators of $\mathrm{SU}(2)$ Lie symmetry group. A comparison with (13) yields

$$
\epsilon=-4 d=16 \widetilde{\mu}^{2} \widetilde{\omega}^{2} \hbar^{2}
$$

and the other structure constants are zero. So, by using (14), we find the Casimir operator of this system as follows:

$$
K=16 \widetilde{\mu}^{4} \widetilde{\omega}^{4} \hbar^{4}-16 \widetilde{\mu}^{4} \widetilde{\omega}^{2} \hbar^{2} \widetilde{H}^{2} .
$$

Regarding the fact that $\gamma=0$ and $\epsilon>0$, the structure function is given by (22). Thus, we can obtain

$$
\Phi(\varepsilon, \mathcal{N})=\frac{1}{4} \widetilde{\mu}^{2}\left(\varepsilon^{2}-(2 \mathcal{N}+2 u-1)^{2} \widetilde{\omega}^{2} \hbar^{2}\right) .
$$

At this stage, by considering the boundary conditions (17) on the structure function $\Phi$ and setting $\mathcal{N}=0$, we find

$$
\begin{aligned}
& u^{\prime}=\frac{1}{2}\left(-\frac{\varepsilon}{\widetilde{\omega} \hbar}+1\right), \\
& u^{\prime \prime}=\frac{1}{2}\left(\frac{\varepsilon}{\widetilde{\omega} \hbar}+1\right) ;
\end{aligned}
$$

the condition $\forall x>0: \Phi(x)>0$ implies that $u^{\prime}$ is the only acceptable solution of the equation. Then, by substituting $u^{\prime}$ and $\mathcal{N}=p+1$ in (41), the energy eigenvalue $\varepsilon$ of the quasiHamiltonian $\widetilde{H}$ reads

$$
\varepsilon=(p+1) \hbar \tilde{\omega}, \quad p=0,2,4, \ldots
$$

Using definition (34), one can obtain

$$
E=-\frac{2 \widetilde{m} \kappa^{2}}{(p+1)^{2} \hbar^{2}}, \quad p=0,2,4, \ldots,
$$

which is the same as (30). Now, the relativistic energy spectrum $\varepsilon_{r}$ of the Klein-Gordon equation with the harmonic oscillator superintegrable potential with spin symmetry (i.e., SVPEM) could be obtained by using (6). Thus, we obtain

$$
\frac{\left(\varepsilon_{r}+M c^{2}\right)\left(\varepsilon_{r}-M c^{2}\right)^{2}}{2 M \omega^{2} c^{2} \hbar^{2}}=(p+1)^{2} .
$$

Now let us substitute 2D Kepler-Coulomb superintegrable potential in the KG equation (5) with SVPEM. So we have

$$
\begin{aligned}
& \left\{c^{2}\left(P_{x}^{2}+P_{y}^{2}\right)+\left(m c^{2}+\mathscr{E}\right) \frac{\kappa}{\sqrt{x^{2}+y^{2}}}\right. \\
& \left.-\left(\mathscr{E}^{2}-m^{2} c^{4}\right)\right\} \psi=0 .
\end{aligned}
$$

By using the Levi-Civita transformation (32), (46) is reduced to [33]

$$
\begin{aligned}
& \left\{c^{2}\left(P_{u_{1}}^{2}+P_{u_{2}}^{2}\right)+4\left(m c^{2}+\mathscr{E}\right) \kappa-4\left(\mathscr{E}^{2}-m^{2} c^{4}\right) u^{2}\right\} \\
& \cdot \psi=0 .
\end{aligned}
$$

By introducing new variables,

$$
\begin{aligned}
4 \kappa & =M c^{2}-\varepsilon_{r}, \\
m c^{2}+\mathscr{E} & =M c^{2}+\varepsilon_{r}, \\
\omega^{2} & =\frac{8\left(m c^{2}-\mathscr{E}\right)}{M},
\end{aligned}
$$

(47) becomes

$$
\begin{gathered}
\left\{c^{2}\left(P_{u_{1}}^{2}+P_{u_{2}}^{2}\right)+\frac{1}{2} M \omega^{2} u^{2}\left(M c^{2}+\varepsilon_{r}\right)\right. \\
\left.-\left(\varepsilon_{r}^{2}-M^{2} c^{4}\right)\right\} \psi=0 .
\end{gathered}
$$

The above equation is the 2-dimensional KG equation for the superintegrable harmonic oscillator with SVPEM. Therefore, (46) and (47) are dual to each other by the Levi-Civita transformation. Consequently, the energy spectra of these two equations will be related to each other by the associated 
transformation. The energy eigenvalues of (49) are obtained in (45). Applying transformations (48) into (45), we find the energy spectrum for (46) as

$$
\mathscr{E}=m c^{2} \frac{-\kappa^{2}+c^{2} \hbar^{2}(p+1)^{2}}{\kappa^{2}+c^{2} \hbar^{2}(p+1)^{2}}
$$

which coincides with the result that was obtained before in (31) by the quadratic algebra approach for the relativistic superintegrable Kepler-Coulomb potential.

4.2. Hyperboloid Superintegrable System. Assume the potential $\widetilde{V}(\boldsymbol{\omega})$ on $\mathrm{HH}(2)$ is

$$
\widetilde{V}(\boldsymbol{\omega})=\mu^{2} \frac{1}{\omega_{3}^{2}}-\nu^{2} \frac{1}{\left(\omega_{1}-\omega_{2}\right)^{2}}+\eta^{2} \frac{\omega_{1}+\omega_{2}}{\left(\omega_{1}-\omega_{2}\right)^{3}},
$$

where $\mu, \nu$, and $\eta$ are positive constants. In this case, the Schrödinger-like equation can admit separation of variables in four coordinates systems and the symmetry operators that commute with the Hamiltonian are given as follows [34].

4.2.1. Equidistant Coordinates. In this system,

$$
\begin{aligned}
& \omega_{1}=\cosh \tau_{1} \cosh \tau_{2}, \\
& \omega_{2}=\cosh \tau_{1} \sinh \tau_{2}, \\
& \omega_{3}=\sinh \tau_{1},
\end{aligned}
$$

where $-\infty<\left(\tau_{1}, \tau_{2}\right)<+\infty$ and the constant of motion is

$$
L_{1}=K_{3}^{2}-2 \nu^{2}\left(\frac{\omega_{1}+\omega_{2}}{\omega_{1}-\omega_{2}}\right)^{2}+2 \eta^{2}\left(\frac{\omega_{1}+\omega_{2}}{\omega_{1}-\omega_{2}}\right)
$$

4.2.2. Horicyclic Coordinates. In this system,

$$
\begin{aligned}
& \omega_{1}=\frac{x^{2}+y^{2}+1}{2 y}, \\
& \omega_{2}=\frac{x^{2}+y^{2}-1}{2 y}, \\
& \omega_{3}=\frac{x}{y},
\end{aligned}
$$

where $y>0,-\infty<x<+\infty$; thus another integral of motion becomes

$$
\begin{aligned}
L_{2}= & \left(K_{2}-M_{1}\right)^{2}-2 \nu^{2}\left(\frac{\omega_{3}}{\omega_{1}-\omega_{2}}\right)^{2} \\
& -2 \mu^{2}\left(\frac{\omega_{1}-\omega_{2}}{\omega_{3}}\right)^{2}+2 \eta^{2} .
\end{aligned}
$$

4.2.3. Elliptic-Parabolic Coordinates. This coordinates system is characterized by

$$
\begin{aligned}
& \omega_{1}=\frac{\cosh ^{2} a+\cos ^{2} \theta}{2 \cosh a \cos \theta}, \\
& \omega_{2}=\frac{\sinh ^{2} a-\sin ^{2} \theta}{2 \cosh a \cos \theta}, \\
& \omega_{3}=\tanh a \tan \theta,
\end{aligned}
$$

where $a>0,-\pi / 2<\theta<+\pi / 2$ and the symmetry operator is given by

$$
\begin{aligned}
L_{3}= & -\left(K_{2}-M_{1}\right)^{2}-K_{3}^{2}+2 \nu^{2} \frac{\left(\omega_{1}+\omega_{2}\right)^{2}+\omega_{3}^{2}}{\left(\omega_{1}-\omega_{2}\right)^{2}} \\
& +2 \mu^{2}\left(\frac{\omega_{1}-\omega_{2}}{\omega_{3}}\right)^{2}-4 \eta^{2} \frac{\omega_{1}}{\omega_{1}-\omega_{2}} .
\end{aligned}
$$

4.2.4. Hyperbolic-Parabolic Coordinates. In this case,

$$
\begin{aligned}
& \omega_{1}=\frac{\cosh ^{2} b+\cos ^{2} \theta}{2 \sinh b \sin \theta}, \\
& \omega_{2}=\frac{\sinh ^{2} b-\sin ^{2} \theta}{2 \sinh b \sin \theta}, \\
& \omega_{3}=\operatorname{coth} b \cot \theta,
\end{aligned}
$$

where $b>0,-\pi / 2<\theta<+\pi / 2$ and the integral of motion is

$$
\begin{aligned}
L_{4}= & \left(K_{2}-M_{1}\right)^{2}-K_{3}^{2}+2 \nu^{2} \frac{\left(\omega_{1}+\omega_{2}\right)^{2}-\omega_{3}^{2}}{\left(\omega_{1}-\omega_{2}\right)^{2}} \\
& -2 \mu^{2}\left(\frac{\omega_{1}-\omega_{2}}{\omega_{3}}\right)^{2}-4 \eta^{2} \frac{\omega_{2}}{\omega_{1}-\omega_{2}} .
\end{aligned}
$$

Now, considering the operators $\left\{L_{1}, L_{2}, L_{3}, L_{4}\right\}$, only two of them are independent:

$$
\begin{aligned}
& L_{3}=-L_{1}-L_{2}, \\
& L_{4}=-L_{1}+L_{2} .
\end{aligned}
$$

However, by constructing the operators $A, B$ as

$$
\begin{aligned}
& A=L_{2}-2 \eta^{2}, \\
& B=L_{1}
\end{aligned}
$$


the quadratic algebra (13) is satisfied. Thus

$[A, B]$

$$
\begin{aligned}
= & -2\left\{K_{3},\left\{K_{2}, M_{1}\right\}\right\}+2\left\{K_{3}, K_{2}^{2}\right\}+2\left\{K_{3}, M_{1}^{2}\right\} \\
& -8\left[\mu^{2}\left(\frac{\omega_{1}-\omega_{2}}{\omega_{3}}\right)^{2}+\nu^{2}\left(\frac{\omega_{3}}{\omega_{1}-\omega_{2}}\right)^{2}\right] K_{3} \\
& +\frac{16 \nu^{2} \omega_{3}}{\left(\omega_{1}-\omega_{2}\right)^{2}}\left(\omega_{2} M_{1}-\omega_{1} K_{2}\right) \\
& +\frac{8 \eta^{2} \omega_{3}}{\omega_{1}-\omega_{2}}\left(K_{2}-M_{1}\right) \\
& +4\left[\eta^{2}+2 \mu^{2}\left(\frac{\omega_{1}-\omega_{2}}{\omega_{3}}\right)^{2}-2 \nu^{2} \frac{1+2 \omega_{3}^{2}}{\left(\omega_{1}-\omega_{2}\right)^{2}}\right]
\end{aligned}
$$

$[A, C]$

$$
\begin{aligned}
= & +8 \hbar^{2} A^{2}+16 \hbar^{2} \eta^{2} A+32 \hbar^{2} \nu^{2} B+64 \widetilde{m} \hbar^{2} v^{2} \widetilde{H} \\
& +16 \hbar^{2} \nu^{2}\left(\hbar^{2}-4 \mu^{2}\right),
\end{aligned}
$$

$[B, C]$

$$
\begin{aligned}
= & -8 \hbar^{2}\{A, B\}+16 \hbar^{4} A-16 \hbar^{2} \eta^{2} B-32 \widetilde{m} \hbar^{2} \eta^{2} \widetilde{H} \\
& +16 \hbar^{2} \eta^{2}\left(\hbar^{2}-2 \mu^{2}\right) .
\end{aligned}
$$

Therefore, the structure constants become

$$
\begin{aligned}
& \alpha=8 \hbar^{2}, \\
& \delta=16 \hbar^{2} \eta^{2}, \\
& \epsilon=32 \hbar^{2} v^{2}, \\
& d=16 \hbar^{4}, \\
& \zeta=64 \widetilde{m} \hbar^{2} v^{2} \widetilde{H}+16 \hbar^{2} v^{2}\left(\hbar^{2}-4 \mu^{2}\right), \\
& z=-32 \widetilde{m} \hbar^{2} \eta^{2} \widetilde{H}+16 \hbar^{2} \eta^{2}\left(\hbar^{2}-2 \mu^{2}\right)
\end{aligned}
$$

and the other structure constants vanish. The Casimir of this system is obtained as

$$
\begin{aligned}
K & =16 \hbar^{2}\left[\eta^{4}\left(8 \mu^{2}-3 \hbar^{2}\right)+4 \nu^{2}\left(2 \widetilde{m}^{2} \widetilde{H}^{2}+2 \mu^{4}\right.\right. \\
& \left.\left.+2 \hbar^{2} \mu^{2}+\widetilde{m} \widetilde{H}\left(4 \mu^{2}-2 \hbar^{2}\right)-\hbar^{4}\right)\right] .
\end{aligned}
$$

Since $\gamma=0$ and $\epsilon>0$, we use (22) to find the structure function

$$
\begin{gathered}
\Phi(E, \mathcal{N})=\frac{1}{16 \hbar^{3} v^{3}}\left[-8 \mu^{2}+(4 \mathcal{N}+4 u-3)(4 \mathscr{N}+4 u\right. \\
-1)]\left\{2 \hbar v \eta^{4}+v^{2} \hbar^{2}\left[4 \sqrt{2}(2 \mathcal{N}+2 u-1) \eta^{2}\right.\right. \\
+(8 m E+(4 \mathscr{N}+4 u-3)(4 \mathscr{N}+4 u-1)) v \hbar]\} .
\end{gathered}
$$

Setting $\mathcal{N}=0$, one can determine parameter $u$ as

$$
\begin{aligned}
& u_{1}=\frac{1}{2}+\frac{\sqrt{8 \mu^{2}+\hbar^{2}}}{4 \hbar}, \\
& u_{2}=\frac{1}{2}-\frac{\sqrt{8 \mu^{2}+\hbar^{2}}}{4 \hbar} .
\end{aligned}
$$

Considering the boundary conditions (17), the structure function (65) should be positive for all values of parameter $u$. Thus by substituting $u_{1}$ and $u_{2}$ in (65) we find out that the structure function is positive just for $u_{1}$. Therefore, by substituting $u_{1}$ and $\mathcal{N}=p+1$ in (65), we obtain energy eigenvalues of the quasi-Hamiltonian as follows:

$$
\begin{array}{r}
E=\frac{-1}{4 \widetilde{m} \nu^{2}}\left\{\eta^{4}+v \eta^{2} \sqrt{2}\left(4 \hbar(p+1)+\sqrt{8 \mu^{2}+\hbar^{2}}\right)\right. \\
\left.+4 v^{2}\left(\mu^{2}+2 \hbar^{2}(p+1)^{2}+\hbar(p+1) \sqrt{8 \mu^{2}+\hbar^{2}}\right)\right\}, \\
p=0,1, \ldots
\end{array}
$$

The energy spectrum obtained by the quadratic algebra approach in (67) is the same as the energy eigenvalues that have been calculated by the approach based on separation of variables and solving the Schrodinger equation in [34]. After some calculations, for this superintegrable system on $\mathrm{HH}(2)$, we find the relativistic energy spectrum of the KG equation with considering SVPEM as follows:

$$
\begin{aligned}
\mathscr{E} & =\frac{ \pm c}{\sqrt{2} v}\left[\eta^{4}-2 m^{2} c^{2} v^{2}+\sqrt{2} v \eta^{2}(4(p+1) \hbar\right. \\
& \left.+\sqrt{8 \mu^{2}+\hbar^{2}}\right)+4 v^{2}\left(\mu^{2}+2(p+1)^{2} \hbar^{2}\right. \\
& \left.\left.+(p+1) \hbar \sqrt{8 \mu^{2}+\hbar^{2}}\right)\right]^{1 / 2} .
\end{aligned}
$$

\section{Conclusion}

The Dirac and Klein-Gordon equations with scalar and vector potentials of equal magnitude (SVPEM) are one of the most interesting areas of quantum physics. In this paper, we investigate the KG equation with the spin symmetry in the presence of superintegrable potentials in two dimensions by means of the quadratic algebra approach which are realized in the deformed oscillator algebra. Indeed the main purpose of this paper is obtaining the relativistic energy spectra of the superintegrable systems. For this reason, we use algebraic methods. The well-known Kepler-Coulomb and isotropic oscillator superintegrable systems and their associated Lie algebras have already been studied. How these two superintegrable systems are connected to each other even in relativistic case is also shown. By applying quadratic algebra approach we find both relativistic and nonrelativistic energy spectra of those systems. So we are trying to use special algebraic approach that has not been investigated before. The relativistic superintegrable harmonic oscillator potential which is dual to the superintegrable Kepler-Coulomb potential and 
a hyperboloid superintegrable potential are studied. The third system is investigated on 2-sheet hyperboloid by quadratic algebra approach for the first time. Solving the Klein-Gordon equation in the presence of this superintegrable system by the mentioned approach is new and the relativistic energy has not been studied before. The transformation of KG equation into a quasi-Hamiltonian (Schrödinger-like equation) is reviewed. Furthermore, the generators of the symmetric groups of the quasi-Hamiltonians and their corresponding quadratic algebras are obtained. Afterwards, we constructed the Casimir operator and structure function of the systems by using the structure constants of the associated quadratic algebras. As a result, energy eigenvalues of the quasi-Hamiltonians are derived. Eventually, we calculated the relativistic energy spectra of the considered superintegrable systems.

\section{Conflict of Interests}

The authors declare that there is no conflict of interests regarding the publication of this paper.

\section{References}

[1] A. Alhaidari, H. Bahlouli, and A. Al-Hasan, "Dirac and KleinGordon equations with equal scalar and vector potentials," Physics Letters A, vol. 349, no. 1-4, pp. 87-97, 2006.

[2] X.-A. Zhang, K. Chen, and Z.-L. Duan, "Bound states of KleinGordon equation and Dirac equation for ring-shaped nonspherical oscillator scalar and vector potentials," Chinese Physics, vol. 14, no. 1, pp. 42-44, 2005.

[3] L.-H. Zhang, X.-P. Li, and C.-S. Jia, "Approximate analytical solutions of the Dirac equation with the generalized Morse potential model in the presence of the spin symmetry and pseudo-spin symmetry," Physica Scripta, vol. 80, no. 3, Article ID 035003, 2009.

[4] F.-L. Zhang and J.-L. Chen, "Dynamical symmetries of the Klein-Gordon equation," Journal of Mathematical Physics, vol. 50, no. 3, Article ID 032301, 2009.

[5] A. de Souza Dutra and M. Hott, "Dirac equation exact solutions for generalized asymmetrical Hartmann potentials," Physics Letters A: General, Atomic and Solid State Physics, vol. 356, no. 3, pp. 215-219, 2006.

[6] J. N. Ginocchio, "U(3) and Pseudo-U(3) symmetry of the relativistic harmonic oscillator," Physical Review Letters, vol. 95, no. 25, Article ID 252501, 3 pages, 2005.

[7] I. Marquette, "Quadratic algebra approach to relativistic quantum Smorodinsky-Winternitz systems," Journal of Mathematical Physics, vol. 52, no. 4, Article ID 042301, 2011.

[8] J. N. Ginocchio, "Relativistic harmonic oscillator with spin symmetry," Physical Review C-Nuclear Physics, vol. 69, no. 3, pp. 034318-1, 2004.

[9] S. Aghaei and A. Chenaghlou, "Solution of the Dirac equation with some superintegrable potentials by the quadratic algebra approach," International Journal of Modern Physics A, vol. 29, no. 6, Article ID 1450028, 2014.

[10] S. Aghaei and A. Chenaghlou, "Quadratic algebra approach to the Dirac equation with spin and pseudospin symmetry for the 4D harmonic oscillator and U(1) monopole," Few-Body Systems, vol. 56, no. 1, pp. 53-61, 2015.
[11] F.-L. Zhang, B. Fu, and J.-L. Chen, "Dynamical symmetry of Dirac hydrogen atom with spin symmetry and its connection with Ginocchio's oscillator," Physical Review A, vol. 78, no. 4, Article ID 040101, 2008.

[12] L. Cheng-Shi, "Classification of all single travelling wave solutions to Calogero-Degasperis-Focas equation," Communications in Theoretical Physics, vol. 48, no. 4, pp. 601-604, 2007.

[13] X.-Q. Hu, G. Luo, Z.-M. Wu, L.-B. Niu, and Y. Ma, "Solving Dirac equation with new ring-shaped non-spherical harmonic oscillator potential," Communications in Theoretical Physics, vol. 53, no. 2, pp. 242-246, 2010.

[14] C. Berkdemir and Y.-F. Cheng, "On the exact solutions of the Dirac equation with a novel angle-dependent potential," Physica Scripta, vol. 79, no. 3, Article ID 035003, 2009.

[15] Z. Yan and G. Jian-You, "The relativistic bound states for a new ring-shaped harmonic oscillator," Chinese Physics B, vol. 17, no. 2, pp. 380-384, 2008.

[16] G.-X. Ju and Z.-Z. Ren, "Supersymmetry and solution of Dirac equation with vector and scalar potentials," Communications in Theoretical Physics, vol. 49, no. 2, pp. 319-326, 2008.

[17] T. Jana and P. Roy, "Shape invariance approach to exact solutions of the Klein-Gordon equation," Physics Letters A: General, Atomic and Solid State Physics, vol. 361, no. 1-2, pp. 55-58, 2007.

[18] E. Olgar, R. Koç, and H. Tütüncüler, "Bound states of the swave equation with equal scalar and vector standard Eckart potential," Chinese Physics Letters, vol. 23, no. 3, pp. 539-541, 2006.

[19] G. Chen, Z.-D. Chen, and Z.-M. Lou, "Exact bound state solutions of the s-wave Klein-Gordon equation with the generalized Hulthén potential," Physics Letters A, vol. 331, no. 6, pp. 374-377, 2004.

[20] G. Chen, Z.-D. Chen, and P.-C. Xuan, "Exactly solvable potentials of the Klein-Gordon equation with the supersymmetry method," Physics Letters A: General, Atomic and Solid State Physics, vol. 352, no. 4-5, pp. 317-320, 2006.

[21] P. Alberto, A. S. D. Castro, and M. Malheiro, "Spin and pseudospin symmetries and the equivalent spectra of relativistic spin-1/2 and spin-0 particles," Physical Review C, vol. 75, no. 4, Article ID 047303, 4 pages, 2007.

[22] C. Daskaloyannis, "Quadratic poisson algebras of two-dimensional classical superintegrable systems and quadratic associative algebras of quantum superintegrable systems," Journal of Mathematical Physics, vol. 42, no. 3, pp. 1100-1119, 2001.

[23] F.-L. Zhang, B. Fu, and J.-L. Chen, "Higgs algebraic symmetry in the two-dimensional Dirac," Physical Review A, vol. 80, no. 5, Article ID 054102, 2009.

[24] Y. I. Granovskii, A. S. Zhedanov, and I. M. Lutzenko, "Quadratic algebra as a 'hidden' symmetry of the Hartmann potential," Journal of Physics A: Mathematical and General, vol. 24, no. 16, pp. 3887-3894, 1991.

[25] Y. I. Granovskii, A. S. Zhedanov, and I. M. Lutsenko, "Quadratic algebras and dynamics in curved spaces. I. Oscillator," Theoretical and Mathematical Physics, vol. 91, no. 2, pp. 474-480, 1992.

[26] D. Bonatsos, C. Daskaloyannis, and K. Kokkotas, "Quantumalgebraic description of quantum superintegrable systems in two dimensions," Physical Review A, vol. 48, no. 5, pp. R3407R3410, 1993.

[27] D. Bonatsos, C. Daskaloyannis, and K. Kokkotas, "Deformed oscillator algebras for two-dimensional quantum superintegrable systems," Physical Review A, vol. 50, no. 5, pp. 3700-3709, 1994. 
[28] C. Daskaloyannis and Y. Tanoudis, "Quadratic algebras for three-dimensional superintegrable systems," Physics of Atomic Nuclei, vol. 73, no. 2, pp. 214-221, 2010.

[29] C. Quesne, "Quadratic algebra approach to an exactly solvable position-dependent mass Schrödinger equation in two dimensions," SIGMA, vol. 3, article 67, 2007.

[30] I. Marquette, "Construction of classical superintegrable systems with higher order integrals of motion from ladder operators," Journal of Mathematical Physics, vol. 51, no. 7, Article ID 037006JMP, 2010.

[31] T. Levi-Civita, "Sur la résolution qualitative du problème restreint des trois corps," Acta Mathematica, vol. 30, no. 1, pp. 305-327, 1906.

[32] E. G. Kalnins, W. Miller Jr., and G. S. Pogosyan, "Coulomboscillator duality in spaces of constant curvature," Journal of Mathematical Physics, vol. 41, no. 5, pp. 2629-2657, 2000.

[33] B. Fu, F.-L. Zhang, and J. Chen, "Connection between Coulomb and harmonic oscillator potentials in relativistic quantum mechanics," Physica Scripta, vol. 81, no. 3, Article ID 035001, 2010.

[34] E. G. Kalnins, W. Miller Jr., Y. M. Hakobyan, and G. S. Pogosyan, "Superintegrability on the two-dimensional hyperboloid. II," Journal of Mathematical Physics, vol. 40, no. 5, pp. 2291-2306, 1999.

[35] C. P. Boyer, E. G. Kalnins, and P. Winternitz, "Completely integrable relativistic Hamiltonian systems and separation of variables in Hermitian hyperbolic spaces," Journal of Mathematical Physics, vol. 24, no. 8, pp. 2022-2034, 1983.

[36] C. Grosche, G. S. Pogosyan, and A. N. Sissakian, "Path-integral approach to superintegrable potentials on the two-dimensional hyperboloid," Physics of Particles and Nuclei, vol. 27, no. 3, pp. 244-278, 1996.

[37] E. G. Kalnins, W. Miller Jr., and G. S. Pogosyan, "Superintegrability on the two-dimensional hyperboloid," Journal of Mathematical Physics, vol. 38, no. 10, pp. 5416-5433, 1997.

[38] W. Miller, Symmetry and Separation of Variables, Encyclopedia of Mathematics and Its Applications, Cambridge University Press, 2012. 

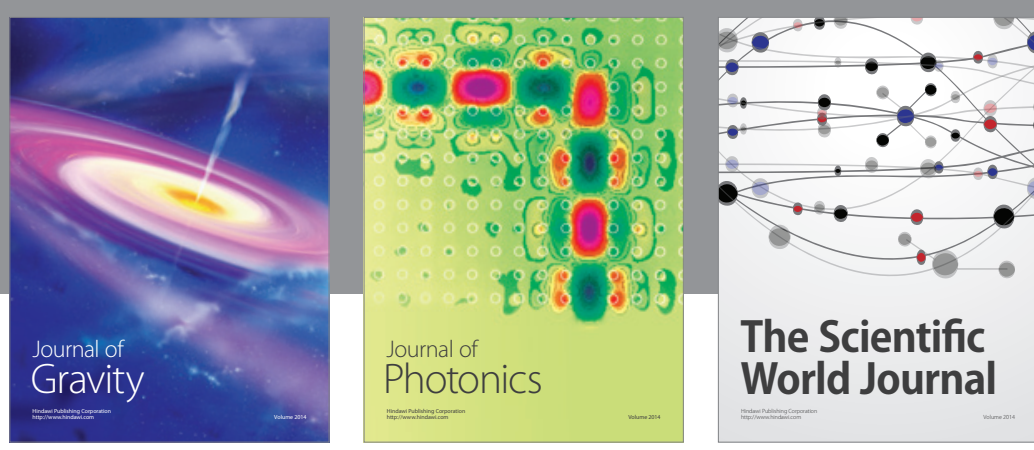

The Scientific World Journal
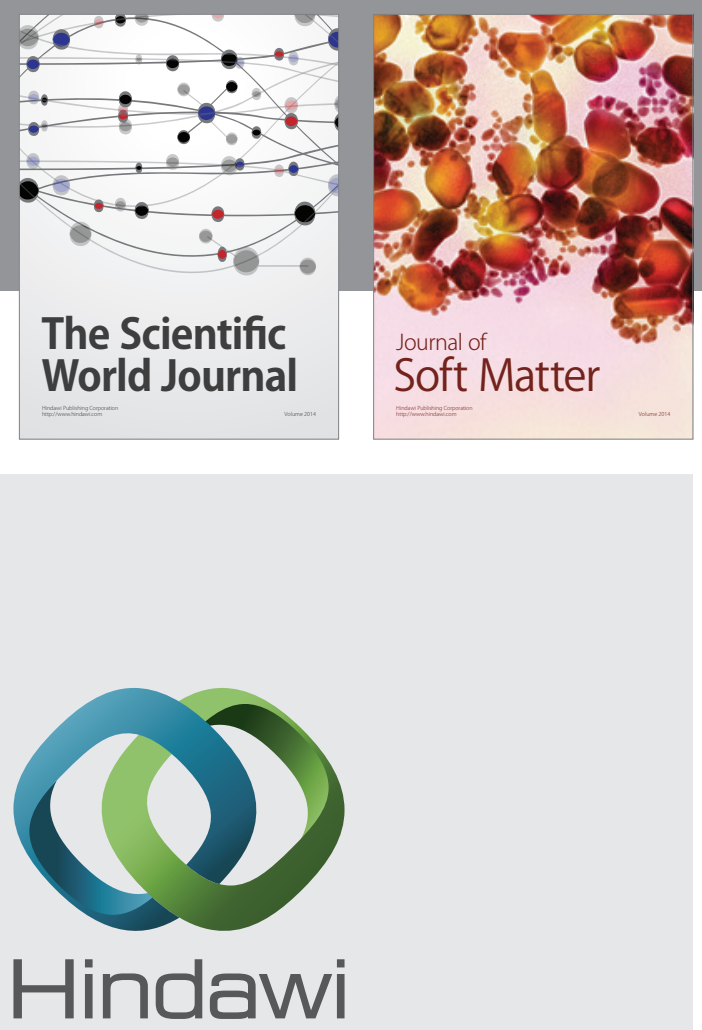

Submit your manuscripts at

http://www.hindawi.com

nternational Journal of

Statistical Mechanics
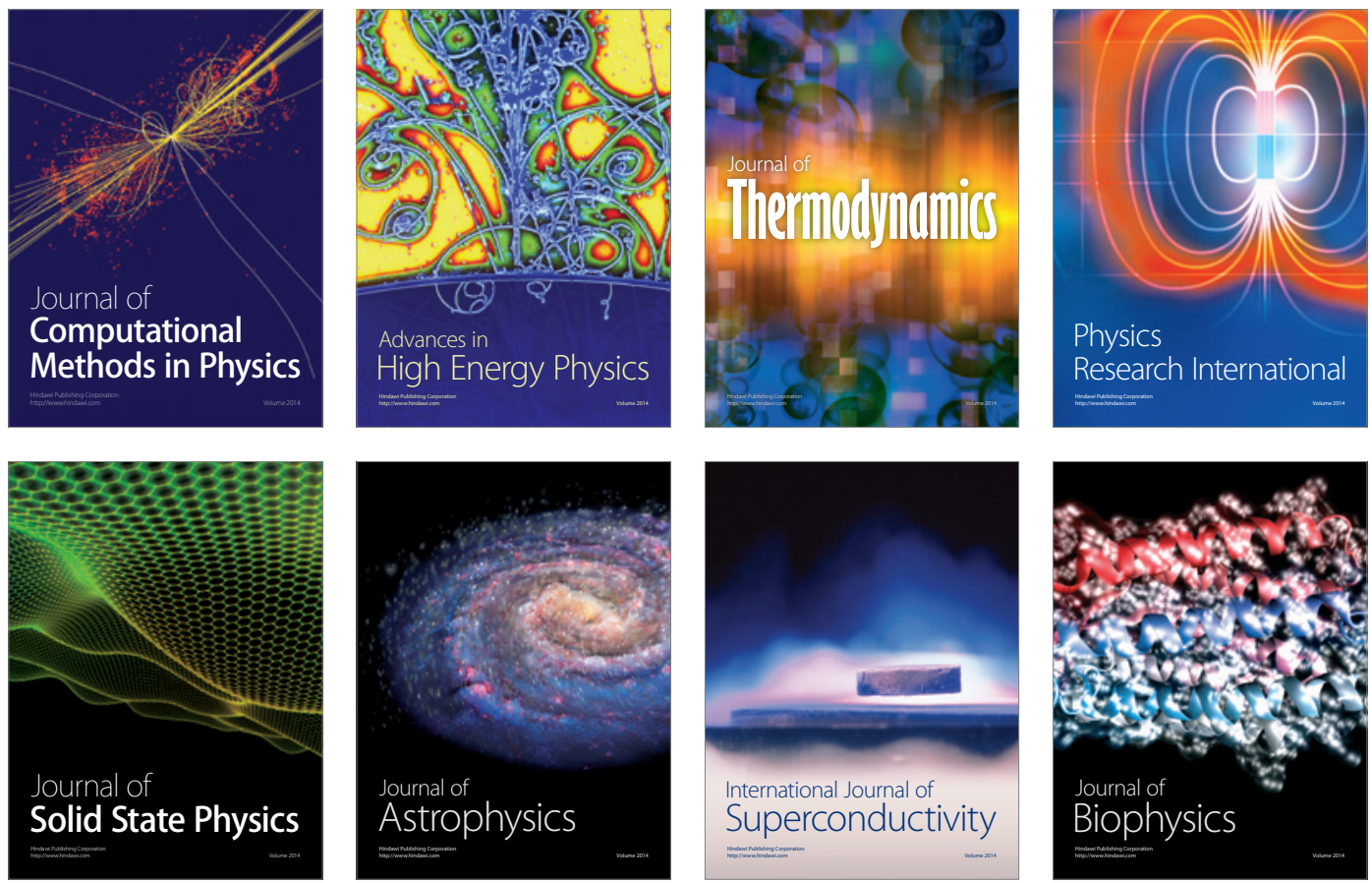
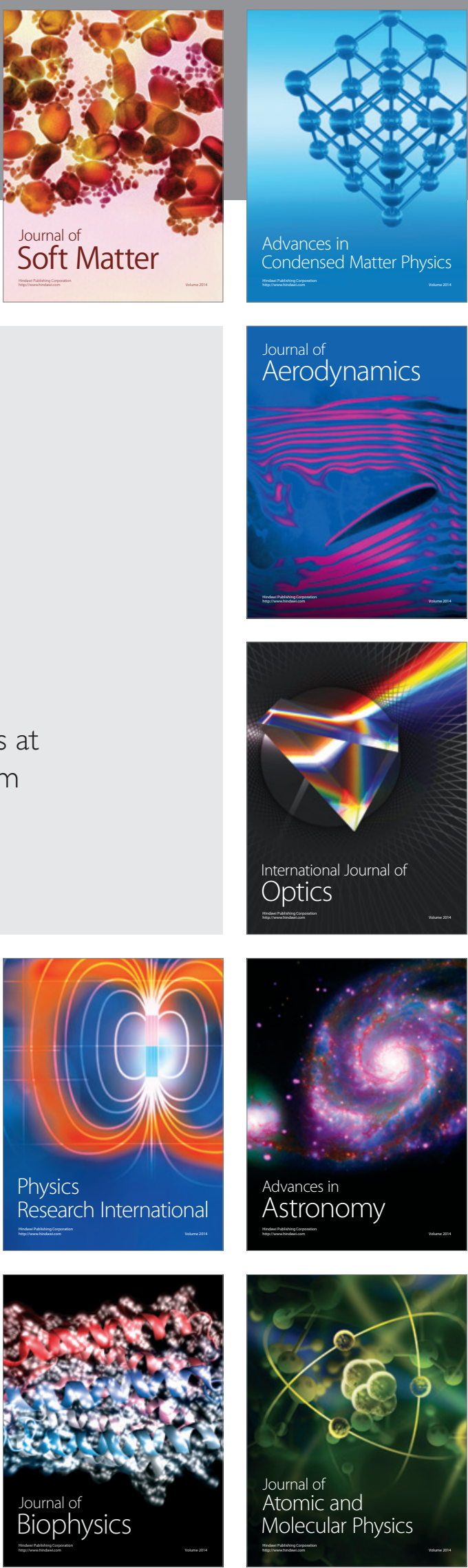